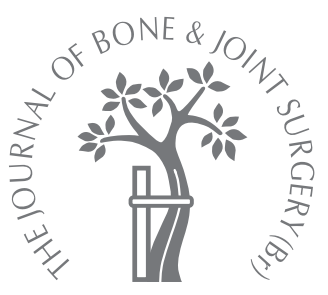

T. Luyckx,

K. Didden,

H. Vandenneucker,

L. Labey,

B. Innocenti,

J. Bellemans

From University

Hospitals UZ,

Pellenberg, Belgium

\title{
Is there a biomechanical explanation for anterior knee pain in patients with patella alta?
}

\author{
INFLUENCE OF PATELLAR HEIGHT ON PATELLOFEMORAL \\ CONTACT FORCE, CONTACT AREA AND CONTACT PRESSURE
}

The purpose of this study was to test the hypothesis that patella alta leads to a less favourable situation in terms of patellofemoral contact force, contact area and contact pressure than the normal patellar position, and thereby gives rise to anterior knee pain.

A dynamic knee simulator system based on the Oxford rig and allowing six degrees of freedom was adapted in order to simulate and record the dynamic loads during a knee squat from $30^{\circ}$ to $120^{\circ}$ flexion under physiological conditions. Five different configurations were studied, with variable predetermined patellar heights.

The patellofemoral contact force increased with increasing knee flexion until contact occurred between the quadriceps tendon and the femoral trochlea, inducing load sharing. Patella alta caused a delay of this contact until deeper flexion. As a consequence, the maximal patellofemoral contact force and contact pressure increased significantly with increasing patellar height $(p<0.01$ ). Patella alta was associated with the highest maximal patellofemoral contact force and contact pressure. When averaged across all flexion angles, a normal patellar position was associated with the lowest contact pressures.

Our results indicate that there is a biomechanical reason for anterior knee pain in patients with patella alta.

Anterior knee pain may be present in as many as $30 \%$ of physically active individuals. ${ }^{1,2}$

Several factors have been associated with anterior knee pain, including patella alta, ${ }^{3-5}$ but the precise mechanism by which this leads to anterior knee pain remains poorly understood.

From a biomechanical point of view, the patellofemoral joint acts as a lever which transmits the force of the quadriceps muscle to the lower leg. ${ }^{6-8}$ The patella increases the moment arm of the quadriceps, especially in late extension, facilitating full extension. ${ }^{9}$ The patellofemoral mechanism is a type of lever which sacrifices force for displacement. The lever arm is relatively short, as the fulcrum is located further away from the load. The load can therefore be displaced over a much greater distance, but the force that needs to be generated is also greater. In the patellofemoral lever system the patella acts as the fulcrum. It has a number of specific characteristics that from a biomechanical point of view make it extremely well suited for its function. In the normal knee, the maximal patellofemoral contact pressure is reached at about $90^{\circ}$ of flexion. The patellofemoral contact area gradually increases with flexion and reaches a maximum at $90^{\circ}$ of flexion, thereby reducing the contact stress in deeper flexion. As the knee flexes, the patellar contact point gradually shifts proximally to an area where the cartilage is thicker. Hence, the patellofemoral joint shows maximal contact area and maximal cartilage thickness in the range of movement where the compressive loads are highest. The delicate balance between the maximal contact area, the maximal thickness of the articulating cartilage and the maximal compressive load can easily be disturbed.

Although some studies ${ }^{10-13}$ have suggested that persons with patella alta may have altered mechanics of knee extension which predispose them to higher joint reaction forces, the literature does not provide a consensus on the precise effect of patella alta on patellofemoral force, contact area and contact pressure.

The recent availability of validated dynamic knee simulators and advances in the analysis of contact force and contact area have now made such studies possible. We have used a third-generation dynamic Oxford knee simulator under physiological loading conditions during a deep knee squat to study the influence of patellar height on patellofemoral contact force, contact area and contact pressure. 

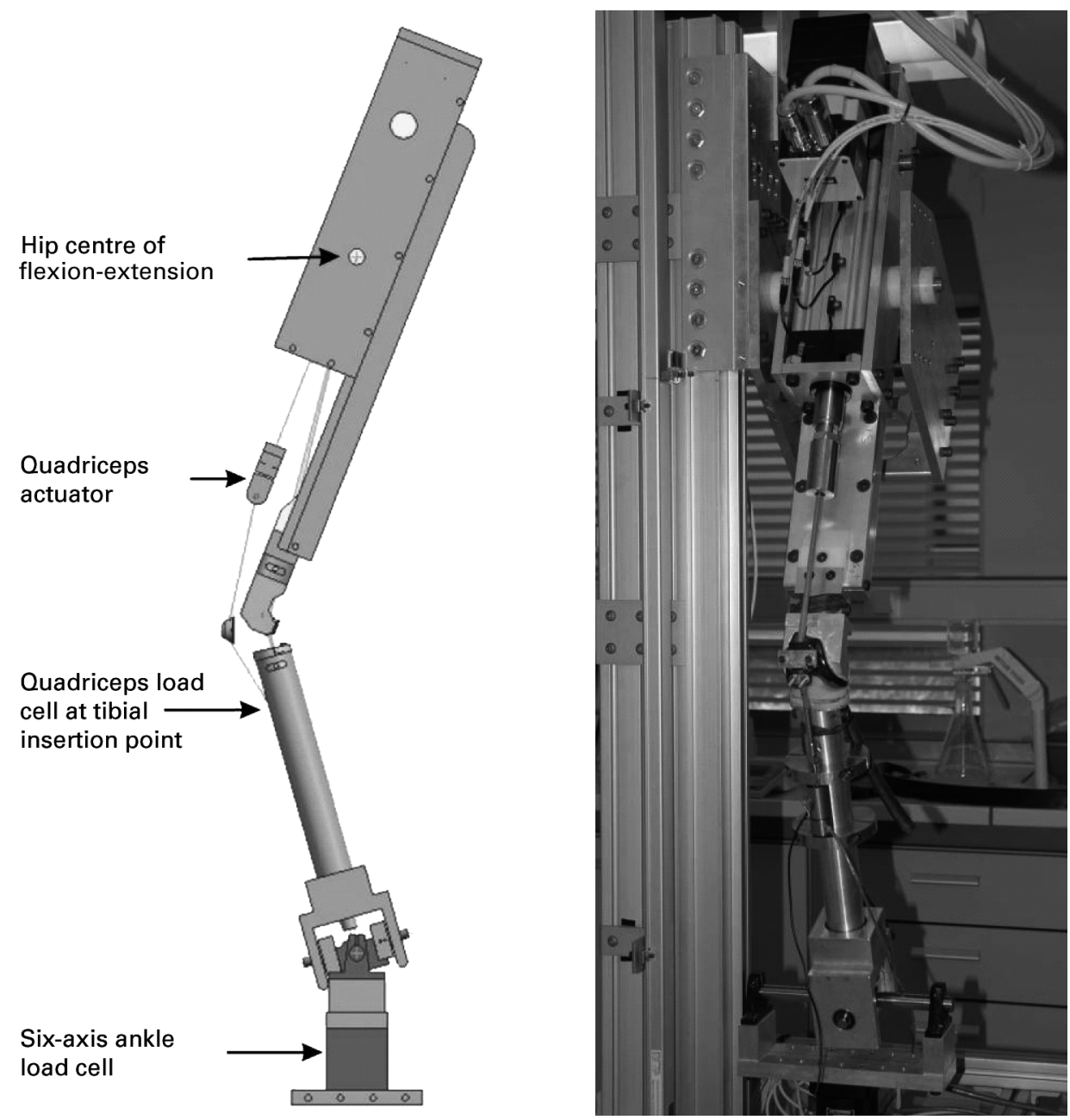

Fig. 1

Schematic drawing and photograph of the knee simulator used for the experiments.

\section{Materials and Methods}

A dynamic knee simulator system based on the Oxford rig was adapted for this study (Fig. 1). It consisted of an upper and lower leg mounted on a frame with a hip and ankle assembly. Two actuators controlled the movement, one moving the hip (vertical sliding) and one pulling the quadriceps. The quadriceps actuator was positioned on the upper leg in a way that reproduced its anatomical location with respect to the moment arms around the knee. Sensors placed in line with the actuators were used to record the patellar tendon force, the ankle forces and moments, and the hip height relative to the ankle.

The knee simulator was designed with $6^{\circ}$ of freedom of movement: $2^{\circ}$ of freedom for movement in the hip joint (flexion/extension and rotation around the transverse axis) and $4^{\circ}$ of freedom for movement in the ankle joint (medial/ lateral translation, flexion/extension, internal/external rotation and abduction/adduction). In this way, the knee was allowed full spatial freedom in order to reproduce its normal kinematics. ${ }^{14}$
An electromechanical system was designed to simulate and record the dynamic loads during a knee squat between $30^{\circ}$ and $120^{\circ}$ of flexion under physiological loading conditions, as described below. A real-time data acquisition and closed feedback system (Labview, National Instruments, Austin, Texas) was used to perform a squat with a certain hip velocity, given as a function of time, while simultaneously applying a quadriceps force on the knee to induce a vertical ankle force. Error feedback from the six-axis ankle load cell was used to control the quadriceps actuator.

Custom-made rigid fixtures simulated the tibia and femur, and were covered with the components of a posteriorly stabilised total knee replacement (TKR). A size 5 femoral component was used with a size 4 baseplate, a size 3 to 4 , $9 \mathrm{~mm}$ thick insert and a $32 \mathrm{~mm}$ resurfacing patellar component (Genesis II, Smith \& Nephew, Memphis, Tennessee). These intermediate size combinations of implants are currently marketed and used clinically. A $6.4 \mathrm{~mm}$ diameter stainless steel cable (Sanlo, Michigan City, Indiana) simulated the patellar and quadriceps tendons. 

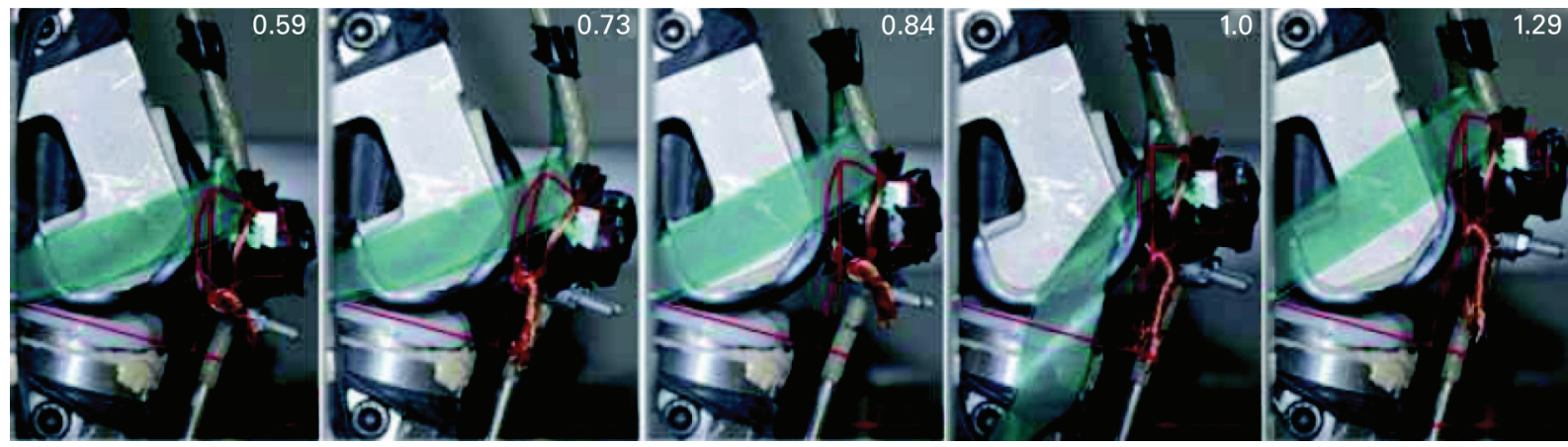

Fig. 2

Illustration of the different positions of the patella. Photographs were used to determine the Blackburne-Peel index for each case. The Blackburne-Peel index is indicated in the right upper corner of each picture.

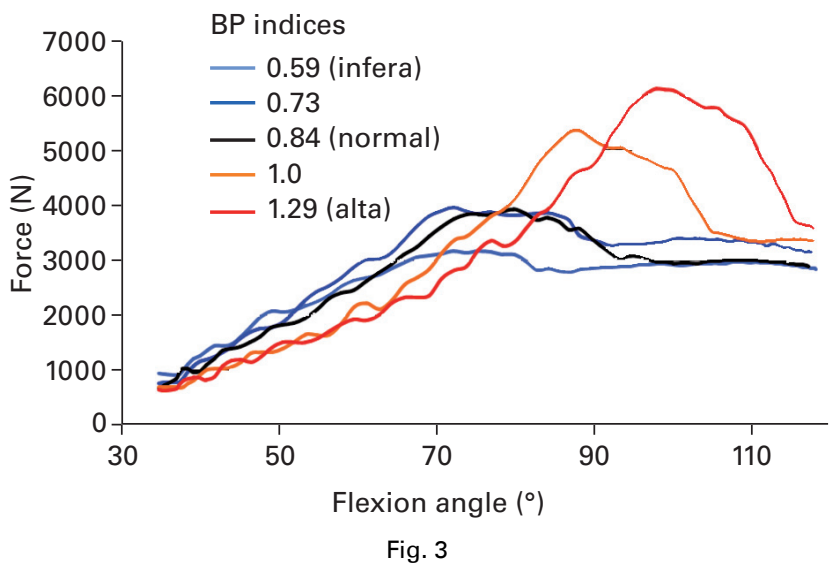

Patellofemoral contact force for the different patellar positions (in terms of Blackburne-Peel index) at $77 \mathrm{lb}$ target ankle load.

A K-Scan 4000/9000 psi sensor (Tekscan,South Boston, Massachusetts) was calibrated according to the manufacturer's instructions. One foot of the sensor was fixed to cover the entire articular surface of the patella using custom wire and adhesive fixtures that did not interfere with the patellofemoral interface. The other foot of the sensor was left unattached. A commercial lubricant was applied at the patellofemoral interface between the sensor and the femoral component to reduce shear forces.

The patellar component was attached to a metal fixture which could be moved along the steel cable that represented the patellar tendon and fixed at different positions, thereby simulating different patellar heights. The Blackburne-Peel index was used to define patellar height. ${ }^{15-17}$ Five different heights were tested: Blackburne-Peel indices of 0.59 (the lowest), 0.73, 0.84 (normal), and 1.0 and 1.29 (patella alta). Lateral photographs were taken in a standardised way at the beginning and end of each experiment, with the knee loaded in $30^{\circ}$ of flexion. The Blackburne-Peel index was then measured on these images (Fig. 2).
The load and movement input curves used for this experiment simulated a single squat from $30^{\circ}$ to $120^{\circ}$ of flexion at a constant hip velocity over a 10 -second interval and with a constant target ankle load. The results of a squat from $120^{\circ}$ to $30^{\circ}$ of extension were similar, but are not discussed here.

For all positions of the patella, each squat was repeated twice at 55,66 and $77 \mathrm{lb}$ target ankle load. In total 30 squats were performed. The quadriceps load, ankle loads and moments, hip position, patellofemoral contact force, contact area and contact pressure were all continuously recorded in a real-time feedback loop.

Statistical analysis. Spearman's correlation coefficient was used to assess the association between the maximal patellofemoral contact force, the contact area and pressure and the patellar height. Statistical analysis used SPSS statistical software (version 16.0; SPSS Inc., Chicago, Illinois) with a significance level of $\mathrm{p}<0.01$.

\section{Results}

For all positions of the patella, the patellofemoral contact force increased with increasing knee flexion. It reached a maximum followed by a decrease (Fig. 3), which coincided with the occurrence of contact between the quadriceps tendon and the femoral trochlea.

In initial flexion $\left(35^{\circ}\right.$ to $\left.70^{\circ}\right)$ lower positions of the patella were associated with higher contact forces. Higher positions were associated with a reduction of the patellofemoral contact forces in this range of flexion. At $60^{\circ}$, for example, there was $780 \mathrm{~N} \mathrm{(41 \% )} \mathrm{less} \mathrm{contact} \mathrm{force} \mathrm{in} \mathrm{the}$ highest position of the patella than in the lowest. Patellar alta was associated with the lowest minimal contact force and the lowest patellar position with the highest minimal contact force. The difference between these was as high as $44 \%(274 \mathrm{~N})$ (Table I).

In deeper flexion $\left(70^{\circ}\right.$ to $\left.120^{\circ}\right)$ patella alta was associated with higher contact forces than with the normal patellar height. Lower positions of the patella were associated with lower contact forces in deeper flexion. There was a direct relationship between the maximal contact 
Table I. Contact forces at different flexion angles, with minimum and maximum contact force for each position of the patella

\begin{tabular}{llllllll}
\hline \multicolumn{7}{l}{ Contact force (N) at } \\
\cline { 2 - 7 } BP $^{*}$ index & Minimum & $\mathbf{4 0}^{\circ}$ & $\mathbf{6 0}^{\circ}$ & $\mathbf{8 0}^{\circ}$ & $\mathbf{1 0 0}^{\circ}$ & $\mathbf{1 2 0}^{\circ}$ & Maximum \\
\hline 0.59 & 903 & 1354 & 2691 & 3098 & 2922 & 2848 & 3169 \\
0.73 & 756 & 1187 & 3004 & 3817 & 3387 & 3147 & 3876 \\
0.84 & 669 & 1085 & 2622 & 3927 & 2944 & 2893 & 3927 \\
1.02 & 668 & 1005 & 2212 & 4119 & 4646 & 3351 & 5365 \\
1.29 & 626 & 813 & 1906 & 3330 & 6101 & 3592 & 6129 \\
\hline
\end{tabular}

* BP, Blackburne-Peel

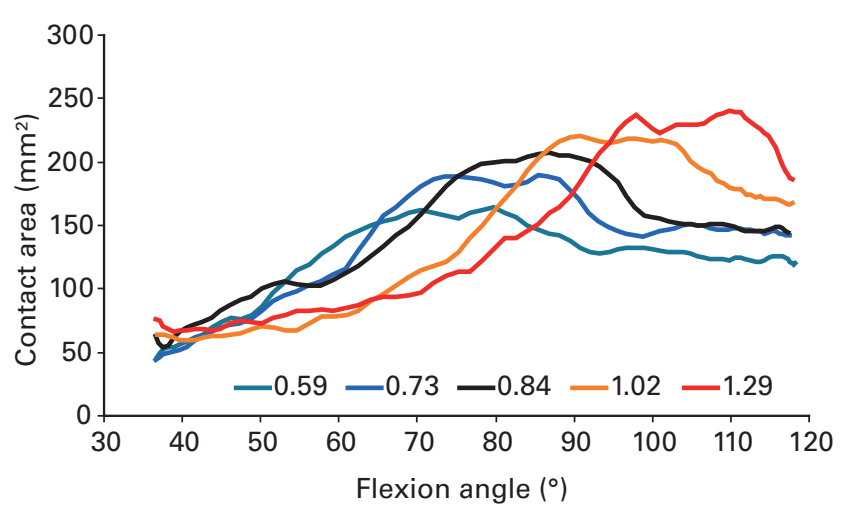

Fig. 4

Experimentally determined patellofemoral contact area for the different patellar positions (in terms of Blackburne-Peel index) at $77 \mathrm{lb}$ target ankle load.

force and patellar height. The higher the position of the patella, the greater the maximal contact force. Patella alta caused the greatest maximal contact force (Table I). The difference in maximal contact force between the highest and the lowest positions of the patella was $3040 \mathrm{~N}$. The flexion angle at which the maximal contact force occurred also increased with increasing patellar height.

Patellar tendon forces measured during the experiment increased with knee flexion until a maximum was reached at $120^{\circ}$ of flexion. No significant differences in patellar tendon force were observed between the different patellar heights.

Total patellofemoral contact area gradually increased with flexion until a maximum was reached, and thereafter decreased (Fig. 4). For the normal patellar height, the contact area reached a maximum at $90^{\circ}$ of flexion (Table II). The maximal total contact area was higher and occurred at deeper angles of flexion for patella alta than for lower positions of the patella $(\mathrm{p}<0.01)$ (Table II). Patella alta was associated with the highest maximal patellofemoral contact area. The difference between the highest and the lowest maximal contact area was $77 \mathrm{~mm}^{2}$. Maximal contact occurred at the transition of patellar support by the trochlea to the femoral condyles.
Table II. Minimum and maximum contact area for each position of the patella, and the flexion angle at which the maximum contact area is seen

\begin{tabular}{|c|c|c|c|}
\hline \multirow[b]{2}{*}{$\mathrm{BP}^{*}$ index } & \multicolumn{2}{|c|}{ Contact area $\left(\mathrm{mm}^{2}\right)$} & \multirow{2}{*}{$\begin{array}{l}\text { Flexion angle at } \\
\text { maximum }\left({ }^{\circ}\right)\end{array}$} \\
\hline & Minimum & Maximum & \\
\hline 0.59 & 44 & 168 & 77 \\
\hline 0.73 & 44 & 192 & 84 \\
\hline 0.84 & 48 & 208 & 89 \\
\hline 1.02 & 58 & 223 & 96 \\
\hline 1.29 & 65 & 245 & 110 \\
\hline
\end{tabular}

* BP, Blackburne-Peel

The Tekscan frames demonstrated a different location of the patellofemoral contact for different heights of the patella in initial flexion $\left(35^{\circ}\right.$ to $\left.70^{\circ}\right)$. The lowest position was associated with the most proximal point of patellofemoral contact. A gradual distal shift of the contact point on the patella was observed with increasing patellar height. Patella alta was associated with the most distal contact point in initial flexion. The recordings of the Tekscan frames at Blackburne-Peel index 0.84 are presented in Figure 5.

The average patellofemoral contact pressures recorded during flexion for the different positions of the patella are presented in Figure 6. Between $30^{\circ}$ and $45^{\circ}$ of flexion, contact pressures were lowest for the higher positions. Lower positions were associated with higher contact pressures in this range of flexion. In deeper flexion $\left(60^{\circ}\right.$ to $\left.105^{\circ}\right)$ contact pressures were highest for the higher positions $(\mathrm{p}<0.01)$. Patella alta was associated with the highest maximal patellofemoral contact pressure. When averaged across all flexion angles, the normal positions of the patella resulted in the lowest contact pressure.

\section{Discussion}

We have examined the effect of the height of the patella on patellofemoral contact force, contact area and contact pressure. As flexion increases beyond $60^{\circ}$, the patellofemoral contact force in a high position of the patella increases further and exceeds the contact force that is recorded with a 

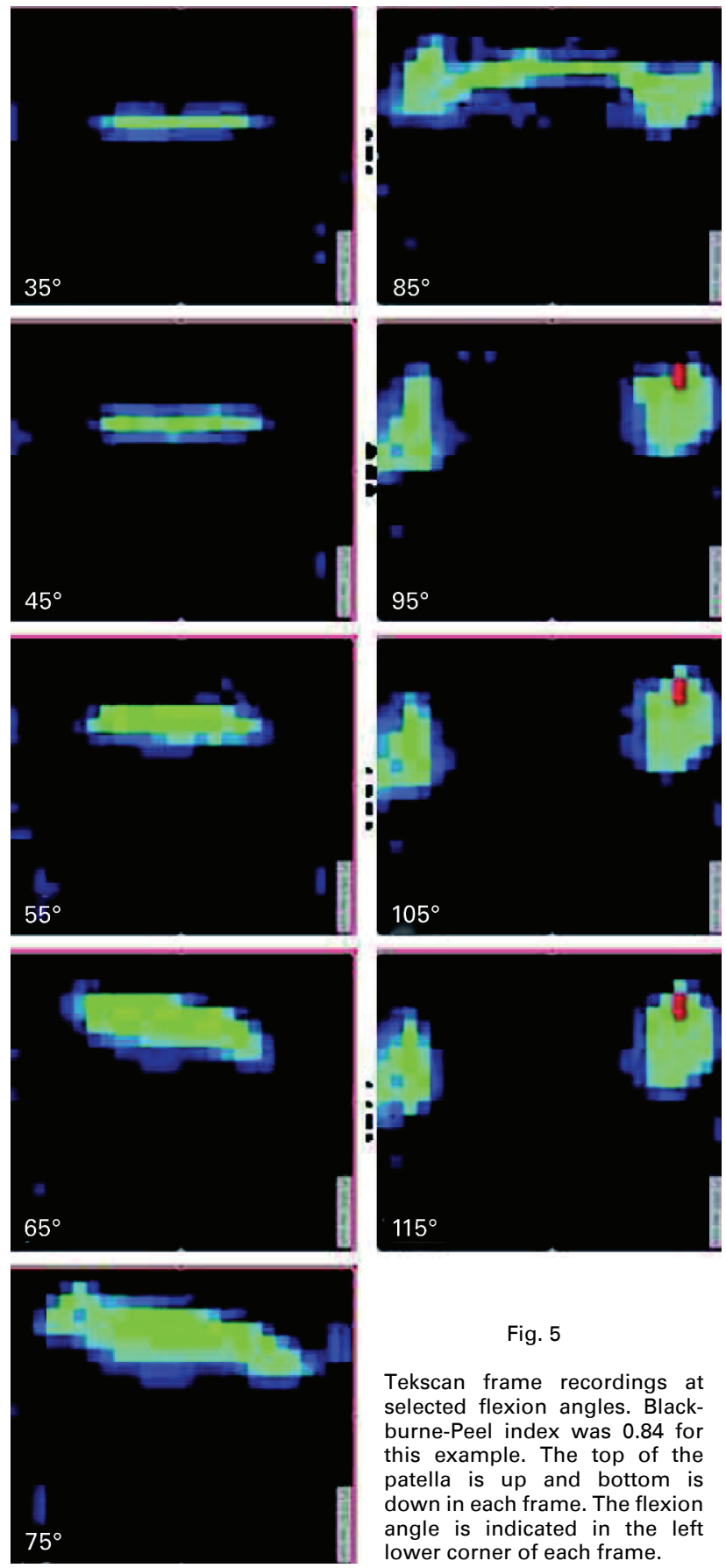

Fig. 5

Tekscan frame recordings at selected flexion angles. Blackburne-Peel index was 0.84 for this example. The top of the patella is up and bottom is down in each frame. The flexion angle is indicated in the left lower corner of each frame.

normal patellar height (Fig. 3). A high position is also associated with a greater maximal contact force. These observations are explained by the tendofemoral contact that occurs beyond a certain degree of flexion. This contact leads to load sharing by the quadriceps tendon and a reduction in contact force on the patellofemoral joint during deeper flexion (Table III). Because a higher position causes the tendofemoral contact to occur at a higher angle of flexion, the patellofemoral contact force is allowed to increase further to a higher maximum. For lower positions the opposite is true. In deeper flexion, patella alta can be considered as a

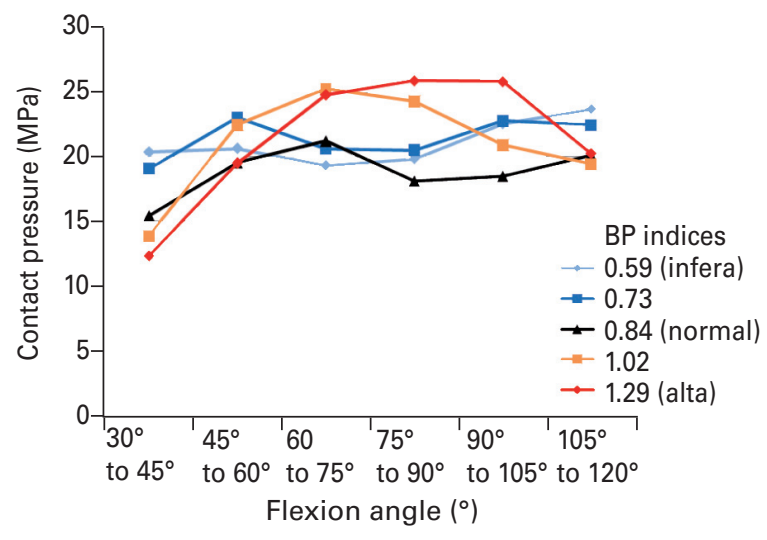

Fig. 6

Average patellofemoral contact pressure for the different patella positions (in terms of Blackburne-Peel index) at $77 \mathrm{lb}$ target ankle load.

\begin{tabular}{|c|c|}
\hline $\mathrm{BP}^{*}$ index & Flexion angle $\left({ }^{\circ}\right)$ \\
\hline 0.59 & 75 \\
\hline 0.73 & 77 \\
\hline 0.84 & 80 \\
\hline 1.02 & 88 \\
\hline 1.29 & 98 \\
\hline
\end{tabular}

* BP, Blackburne-Peel

biomechanical disadvantage compared to normal patellar height. Huberti and Hayes ${ }^{18}$ have described the effect of tendofemoral contact. According to them, at $120^{\circ}$ of flexion this contact would support up to half of the total patellofemoral contact force. In patella infera Meyer et a ${ }^{19}$ observed load sharing by the tendofemoral contact of up to $80 \%$ of the contact force.

Our data are consistent with those of Singerman, Davy and Goldberg, ${ }^{20}$ who suggested that the patellofemoral contact force depended on patellar height. However, they observed no reduction in contact force after tendofemoral contact had occurred.

We have also demonstrated that in initial flexion the contact forces on patella alta were smaller than in lower positions of the patella. When the knee is flexed less than $60^{\circ}$, patella alta reduces the contact force, and there is a direct correlation between this reduction and the patellar height.

The biomechanical function of the patella in the extensor mechanism explains these differences in initial flexion. The patella contributes significantly to the torque in knee extension by increasing the lever arm of the quadriceps. The patella also transmits the forces from the quadriceps tendon to the patellar tendon. For this, it is free to pivot around its contact point with the femoral trochlea in the sagittal 
plane, acting as a balancing beam. Because the contact point with the femur lies distally on the patella in initial flexion, the effective moment arm of the quadriceps tendon is greater than that of the patellar tendon. ${ }^{10,21,22}$ So, in initial flexion, a smaller quadriceps force is needed to generate a certain patellar tendon force. ${ }^{23}$ As patella alta causes a more distal contact point in initial flexion, it reinforces this effect and creates a more efficient knee extensor mechanism.

The patellofemoral contact force is the sum of the patellar tendon force and the quadriceps tendon force. In our experiment, the patellar tendon force was measured and no significant differences were observed at the different heights of the patella. The reduction in patellofemoral contact force in patella alta is therefore explained by a reduced quadriceps tendon force due to the more efficient extensor mechanism of the knee.

With respect to this, our data are consistent with the previous findings of Ward et $\mathrm{al}^{13}{ }^{13}$ who were able to demonstrate in an MRI study that individuals with patella alta had a significantly larger quadriceps effective moment arm between $0^{\circ}$ and $60^{\circ}$ of knee flexion. Based on these findings, they suggested that patients with patella alta may experience less patellofemoral contact force to overcome the same knee flexion moment in the range of $0^{\circ}$ to $60^{\circ}$. Our study confirms this hypothesis by direct in vitro measurement of the patellofemoral contact force during a deep knee squat.

The study has a number of limitations. It was undertaken using an artificial implant which allowed adjustment of the height of the patella easily without destroying the knee. Since the knee simulator was designed with $6^{\circ}$ of freedom of movement, the knee was allowed its full spatial freedom so that its kinematics could be closely reproduced. ${ }^{14}$ The study was conducted on a morphologically normal knee but patella alta is a component of complex patellofemoral dysplasia in which other factors, such as malalignment and lateral tilt, may influence contact stresses. A recent MRI study showed that patella alta was associated with more lateral displacement and lateral tilt at $0^{\circ}$ of flexion, and with a smaller contact area between $0^{\circ}$ and $60^{\circ}$ of flexion. ${ }^{24}$ However, no correlation could be found between malalignment and the reduced contact areas. The authors concluded that these observations warrant careful reconsideration of the previously-accepted cause-and-effect relationship between patellofemoral malalignment and reduced patellofemoral contact area. A limitation of our study was that we were unable to take into account the three dimensional behaviour of cartilage at contact and the co-activation of surrounding muscles which occurs during knee flexion and are known to play an important role in patellofemoral kinematics. Nevertheless, we believe our study indicates that, from a biomechanical point of view, patella alta is a less favourable situation in terms of contact force and contact pressure.

With regard to the patellofemoral contact area, we found that for a normal patellar height, the contact area reached a maximum around $90^{\circ}$ of flexion and decreased thereafter (Table II). This corresponded with the transition from one central contact area to two separate smaller areas as the patella progressed from the femoral trochlea onto the two condyles at the intercondylar notch (Fig. 5).

A higher position of the patella corresponded with a lower contact area in initial flexion, whereas a lower position was associated with a higher contact area for the low angles of flexion. These findings are consistent with previous observations. ${ }^{24,25}$ Ward et $\mathrm{al},{ }^{24}$ in a recent MRI study, concluded that a vertical position of the patella was negatively associated with the contact area between $0^{\circ}$ and $60^{\circ}$ of knee flexion.

Our study also demonstrated that the maximum patellofemoral contact area gradually increased with increasing patellar height. This can be explained by the greater forces observed in deep flexion for these higher positions. A higher patellofemoral contact force causes more deformation of the articulating surface, resulting in an increased contact area. These data are consistent with previous in vivo MRI studies showing a greater contact area in weightbearing than in non-weight-bearing for a given angle of flexion, indicating that contact area increases with increasing load. ${ }^{26,27}$

In initial flexion, contact pressures were the lowest for the higher positions of the patella, whereas lower positions resulted in a higher contact pressure (Fig. 6). In deeper flexion, however, the contact pressure on the higher patellae was the greatest. The maximal patellofemoral contact pressure increased with increasing patellar height. When averaged across all flexion angles, the normal height of the patella demonstrated the lowest contact pressure and can therefore be considered to be the most favourable.

Patella alta demonstrated the highest maximal patellofemoral contact force and the highest contact pressure when averaged over the whole range of movement. We believe that this is a consequence of the delay in tendofemoral contact and that this may provide a biomechanical explanation for anterior knee pain in patients with patella alta.

No benefits in any form have been received or will be received from a commercial party related directly or indirectly to the subject of this article.

\section{References}

1. Jordaan G, Schwellnus MP. The incidence of overuse injuries in military recruits during basic military training. Mil Med 1994;159:421-6.

2. Levine J. Chondromalacia patellae. Phys Sportsmed 1979;7:40-3.

3. Kujala UM, Osterman K, Kvist M, Aalto T, Friberg O. Factors predisposing to patellar chondropathy and patellar apicitis in athletes. Int Orthop 1986;10:195-200.

4. Lancourt JE, Christi JA. Patella alta and patella infera: their etiological role in patellar dislocation, chrondromalacia, and apophysitis of the tibial tubercle. J Bone Joint Surg [Am] 1975;57-A:1112-15.

5. Kannus PA. Long patellar tendon: radiographic sign of patellofemoral pain syndrome: a prospective study. Radiology 1992;185:859-63.

6. Bellemans J. Biomechanics of anterior knee pain. Knee 2003;10:123-6

7. Hungerford DS, Barry M. Biomechanics of the patellofemoral joint. Clin Orthop 1979;144:9-15.

8. Hehne HJ. Biomechanics of the patellofemoral joint and its clinical relevance. Clin Orthop 1990;258:73-85. 
9. Grelsamer RP, Weinstein CH. Applied biomechanics of the patella. Clin Orthop 2001;389:9-14.

10. Yamaguchi GT, Zajac FE. A planar model of the knee joint to characterize the knee extensor mechanism. J Biomech 1989;22:1-10.

11. Hirokawa S. Three-dimensional mathematical model analysis of the patellofemoral joint. J Biomech 1991;24:659-71.

12. Meyer SA, Brown TD, Pedersen DR, Albright JP. The effects of patella infera on patellofemoral contact stress. Trans Orthop Res Soc 1993;18:303.

13. Ward SR, Terk MR, Powers CM. Influence of patella alta on knee extensor mechanics. J Biomech 2005;38:2415-22.

14. Zavatsky AB. A kinematic-freedom analysis of a flexed-knee-stance testing rig. $J$ Biomech 1997;20:277-80.

15. Blackburne JS, Peel TE. A new method of measuring patellar height. J Bone Joint Surg [Br] 1977;59-B:241-2.

16. Berg EE, Mason SL, Lucas MJ. Patellar height ratios: a comparison of four measurement methods. Am J Sports Med 1996;24:218-21.

17. Rogers BA, Thornton-Bott P, Cannon JR, Briggs TW. Interobserver variation in the measurement of patellar height after total knee arthroplasty. J Bone Joint Surg [Br] 2006;88-B:484-8.

18. Huberti HH, Hayes WC. Patellofemoral contact pressures: the influence of q-angle and tendofemoral contact. J Bone Joint Surg [Am] 1984;66-A:715-24.
19. Meyer SA, Brown TD, Pedersen DR, Albright JP. Retropatellar contact stress in simulated patella infera. Am J Knee Surg 1997;10:129-38.

20. Singerman R, Davy DT, Goldberg VM. Effects of patella alta and patella infera on patellofemoral contact forces. J Biomech 1994;27:1059-65.

21. Grood ES, Suntay W, Noyes FR, Butler DL. Biomechanics of the knee-extension exercise: effect of cutting the anterior cruciate ligament. J Bone Joint Surg [Am] 1984;66-A:725-34.

22. van Eijden TM, Kouwenhoven E, Verberg J, Weijus WA. A mathematical model of the patellofemoral joint. J Biomech 1986;19:219-29.

23. Huberti HH, Hayes WC, Stone JL, Shybut GT. Force ratios in the quadriceps tendon and ligamentum patellae. J Orthop Res 1984;2:49-54.

24. Ward SR, Terk MR, Powers CM. Patella alta: association with patellofemora alignment and changes in contact area during weight-bearing. J Bone Joint Surg [Am] 2007;89-A:1749-55

25. Salsich GB, Ward SR, Terk MR, Powers CM. In vivo assessment of patellofemoral joint contact area in individuals who are pain free. Clin Orthop 2003;417:277-84.

26. Besier TF, Draper CE, Gold GE, Beaupre GS, Delp SL. Patellofemoral joint contact areas increase with knee flexion and weight-bearing. J Orthop Res 2005;23:345-50.

27. Gold GE, Besier TF, Draper CE, Asakawa DS, Delp SL. Weight-bearing MRI of patelllofemoral joint cartilage contact area. J Magn Reson Imaging 2004;20:526-30. 